\title{
Vitamin D and DDX4 regulate the proliferation and invasion of ovarian cancer cells
}

\author{
YOUGUO CHEN ${ }^{1,2^{*}}$, ZIQIAN SUN $^{2 *}$, JINGBO XU $^{2}$, PEIFANG WANG $^{1}$, JUAN TANG $^{1}$, \\ XIAORUI SHI ${ }^{1}$, JINGJING LIU ${ }^{1}$, FANGFANG REN ${ }^{1}$ and LAN XU ${ }^{1}$ \\ ${ }^{1}$ Department of Biochemistry and Molecular Biology, Soochow University Medical College; ${ }^{2}$ Department of Obstetrics \\ and Gynecology, The First Affiliated Hospital of Soochow University, Suzhou, Jiangsu 215123, P.R. China
}

Received March 29, 2017; Accepted January 19, 2018

DOI: $10.3892 / \mathrm{ol} .2018 .8718$

\begin{abstract}
Ovarian cancer is one of the most commonly occurring types of cancer and one of the most common causes of cancer-associated mortality in women. Diagnosis of ovarian cancer at an early stage is difficult due to the lack of specific symptoms. In the present study, it is demonstrated that active vitamin $\mathrm{D}$ treatment prohibited the proliferation and invasion of ovarian cancer cells, and the expression level of a germ cell specific marker DEAD (Asp-Glu-Ala-Asp)-box helicase 4 (DDX4), which is overexpressed in ovarian cancer, was downregulated by active vitamin D treatment. Knockdown of DDX4 by siRNA could also suppress the invasive ability of ovarian cancer cells. Therefore, DDX4 may be considered as a diagnostic marker of ovarian cancer, and vitamin D may be a candidate drug for ovarian cancer therapy.
\end{abstract}

\section{Introduction}

Ovarian cancer is one of the most common causes of cancer-associated mortality in women (1). The majority of patients with ovarian cancer are diagnosed at an advanced stage, as there are no reliable symptoms for early diagnosis. Even at advanced stages of disease, signs and symptoms remain nonspecific (2). Upon diagnosis, ovarian cancer has often already metastasized to the uterus, peritoneum or other organs in the pelvic cavity $(3,4)$. Radical surgery and adjuvant chemotherapy are common modes of treatment for ovarian cancer $(5,6)$.

Correspondence to: Dr Lan Xu or Dr Fangfang Ren, Department of Biochemistry and Molecular Biology, Soochow University Medical College, 199 Renai Road, Suzhou, Jiangsu 215123, P.R. China

E-mail: xulan@suda.edu.cn

E-mail: ffren@suda.edu.cn

*Contributed equally

Abbreviations: DEAD, Asp-Glu-Ala-Asp; DDX4, DEAD-box helicase 4; CCK-8, Cell Counting Kit-8; RT-qPCR, reverse transcription-quantitative polymerase chain reaction

Key words: vitamin D, Asp-Glu-Ala-Asp box polypeptide, ovarian cancer, SKOV3 and OVCAR3 cells, invasion
Vitamin D has been reported to inhibit the recurrence and distant metastasis of 19 types of cancer, of which ovarian cancer is one $(7,8)$. Furthermore, a previous study demonstrated that mortality rates of ovarian cancer are lower in areas with higher levels of ultraviolet-B (UVB) radiation (9). An association between vitamin D deficiency and the occurrence of ovarian cancer has been suggested (10).

The germ cell-specific marker DEAD (Asp-Glu-Ala-Asp)box helicase 4 (DDX4), which is the human ortholog of the Drosophila vasa gene, encodes a member of the DEAD-box family of ATP-dependent RNA helicases (11).DDX4 is expressed solely in germ cells, including oocytes and spermatocytes, and has been reported to serve a central role in several aspects of germ cell development (11). A previous study demonstrated that DDX4 is overexpressed in ovarian cancer (12).

The present study demonstrated that the proliferative and invasive capacities of ovarian cancer cells were suppressed by active vitamin D. Vitamin D treatment downregulated the expression level of DDX4, and knockdown of DDX4 reduced the invasive ability of ovarian cancer cells.

\section{Materials and methods}

Cell culture. The ovarian epithelial carcinoma SKOV3 and OVCAR3 cell lines were purchased from the Cell Bank of Type Culture Collection of Chinese Academy of Sciences (Shanghai, China). Cells were cultured in RPMI-1640 medium (Gibco; Thermo Fisher Scientific, Inc., Waltham, MA, USA) supplemented with $10 \%$ fetal bovine serum (FBS; Zhejiang Tianhang Biotechnology Co., Ltd., Sijiqing, China) and 1\% penicillin-streptomycin (Beyotime Institute of Biotechnology, Haimen, China), and incubated at $37^{\circ} \mathrm{C}$ with $5 \% \mathrm{CO}_{2}$. When cultured to $70 \%$ confluence, SKOV3 or OVCAR3 cells were treated with active vitamin D (catalog no. D1530; Sigma-Aldrich; Merck KGaA, Darmstadt, Germany).

Small interfering RNA (siRNA) treatment. The siRNAs targeting DDX4 were designed by GenePharma Co., Ltd. (Shanghai, China) and transfected (100 pmol siRNA for each well containing $2 \times 10^{5}$ cells) into SKOV3 or OVCAR3 cells using Lipofectamine $^{\circledR} 2000$ (Thermo Fisher Scientific, Inc.) according to the manufacturer's protocol. RNA was extracted 3 or 5 days after transfection for the following experiments. The sequences 
for DDX4-712 (7656; GenePharma Co., Ltd.) were as follows; sense, 5'-GGAAGUGAACGAGGUGGUUTT-3' and antisense, 5'-AACCACCUCGUUCACUUCCTT-3'. The sequences for DDX4-121 (7654; GenePharma Co., Ltd.) were as follows; sense, 5'-GCAGAAAUCAACCCUCAUATT-3' and antisense, 5'-UAUGAGGGUUGAUUUCUGCTT-3'. The sequences for the negative control (7653; GenePharma Co., Ltd.) were as follows: Sense, 5'-UUCUCCGAACGUGUCACGUTT-3', and antisense, 5'-ACGUGACACGUUCGGAGAATT-3'.

Cell Counting Kit-8 (CCK-8) assay. The relative cell number was measured using a CCK- 8 assay. Briefly, cells were cultured in 96-well plates at a density of $1 \times 10^{3}$ cells/well for $24 \mathrm{~h}$, then treated with $0,25,50,100,250$ or $400 \mu \mathrm{l} / \mathrm{ml}$ active vitamin D for $72 \mathrm{~h}$. Subsequently, $10 \mu \mathrm{l} \mathrm{CCK}-8$ dye (Beyotime Institute of Biotechnology) was added to each well, according to the manufacturer's protocol. The plates were read using a microplate reader at a wavelength of $450 \mathrm{~nm}$. Relative cell number was represented by the absorbance value relative to that of the untreated control cells.

RNA extraction and reverse transcription-quantitative polymerase chain reaction ( $R T-q P C R)$. Total RNA was extracted from SKOV3 or OVCAR3 cells using TRIzol (Thermo Fisher Scientific, Inc.), according to the manufacturer's protocol. cDNA was synthesized using a ReverTraAce qPCR kit (Toyobo Life Science, Osaka, Japan, Japan), according to the manufacturer's protocol. PCR was performed using an ABI PRISM 7500 system (Thermo Fisher Scientific, Inc.), and the thermocycling conditions were as follows: $95^{\circ} \mathrm{C}$ for $60 \mathrm{sec}$, 40 cycles of $95^{\circ} \mathrm{C}$ of $15 \mathrm{sec}$ and $60^{\circ} \mathrm{C}$ of $60 \mathrm{sec}$. The primer sequences for DDX4 are as follows: Forward, CCAGAGGGC TGGATATTGAA, and reverse, GCCAGTATTCCCACA ACGAC. The primer sequences for GAPDH are as follows: Forward, AATCCCATCACCATCTTCCA and reverse, AAA TGAGCCCCAGCCTTCT. The $\triangle \mathrm{Cq}=\mathrm{Cq}_{\text {gene }}-\mathrm{Cq}_{\text {reference }}$ calculation was adopted to scale the relative levels of gene expression, and $2^{-\Delta \Delta C q}$ method was used to calculate the fold change of gene expression (13). qPCR was performed in duplicate for 3 independent groups of treated cells.

Western blotting. SKOV3 and OVCAR3 cells extracts were lysed with mammalian protein extraction reagent (CWBIO, Beijing, China) supplemented with $1 \%$ protease inhibitors (CWBIO) at $4^{\circ} \mathrm{C}$ for $30 \mathrm{~min}$. The suspension was then centrifuged at $10,000 \mathrm{xg}$ for $10 \mathrm{~min}$ at $4^{\circ} \mathrm{C}$. The supernatant was collected and the protein concentration was determined using a BCA protein quantitation kit (Pierce; Thermo Fisher Scientific, Inc.), according to the manufacturer's protocol. Proteins $(10 \mu \mathrm{g})$ were separated by $12 \%$ SDS-PAGE and transferred into nitrocellulose membranes (Merck KGaA, Darmstadt, Germany). The membranes were blocked with $5 \%$ non-fat milk in Tris buffered saline with $0.5 \%$ Tween-20 for $1 \mathrm{~h}$ at room temperature, then incubated with the following primary antibodies overnight at $4^{\circ} \mathrm{C}$ : DDX4 (dilution 1:1,000, cat. no. ab13840; Abcam, Cambridge, UK) and GAPDH (dilution 1:1,000, cat. no. AG019-1; Beyotime Institute of Biotechnology, Haimen, China). The membranes were then incubated with a horseradish peroxidase-conjugated anti-rabbit secondary antibody (dilution, 1:1,000, cat. no. SA00001-2; ProteinTech

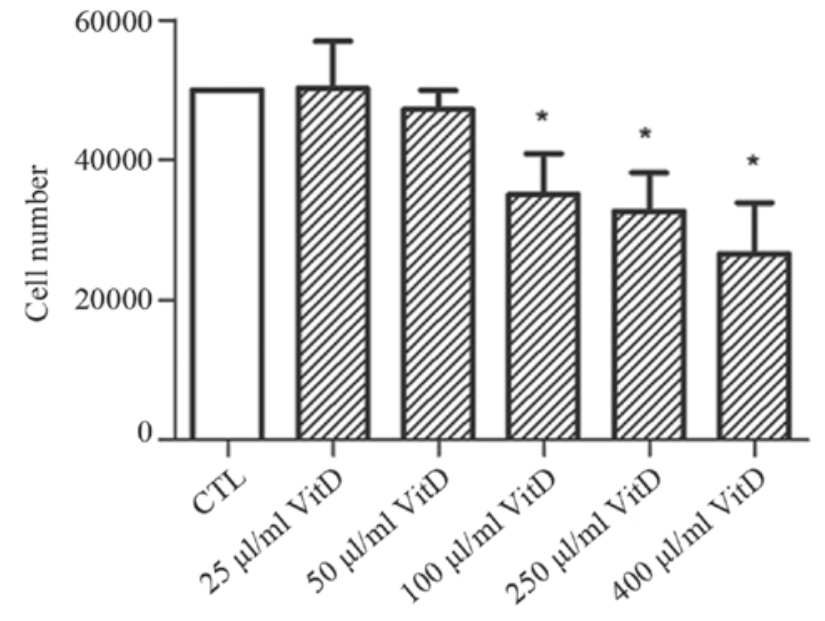

Figure 1. Active vitamin D inhibits the proliferation of SKOV3 and OVCAR3 cells. SKOV3 or OVCAR3 cells were treated with the indicated concentrations of active vitamin $\mathrm{D}$, and proliferation was quantified relative to the untreated control cells. ${ }^{*} \mathrm{P}<0.05$, compared with control. CTL, control; VitD, active vitamin $\mathrm{D}$.

Group, Inc., Chicago, IL, USA) for DDX4 and anti-mouse for GAPDH (dilution 1:1,000, cat. no. AF0006, Beyotime Institute of Biotechnology) for $2 \mathrm{~h}$ at room temperature and visualized by chemiluminescence using an eECL western blot kit (cat. no. CW0049; CWBIO) and western enhanced chemiluminescence substrates (cat. nos. 102030838 and 102030839; Bio-Rad Laboratories, Inc., Hercules, CA, USA). The western blotting results were quantified using ImageJ software (version 1.48; National Institutes of Health, Bethesda, MD, USA).

Transwell invasion assay. The transwell apparatus was assembled using $8-\mu \mathrm{m}$ pore Transwell inserts (Corning Incorporated, Corning, NY, USA) in 24-well plates. Each insert were coated with $100 \mu 1$ Matrigel (diluted in PBS, 1:1). A total of $1 \times 10^{5}$ SKOV3 or OVCAR3 cells were seeded onto the insert and cultured with $250 \mu 1$ RPMI-1640 medium supplemented with $1 \%$ FBS, while the lower chambers contained $500 \mu \mathrm{l}$ RPMI-1640 supplemented with 10\% FBS. Subsequent to incubation for 3 or 5 days, the cells in the upper chambers were removed carefully using cotton swabs, and cells that traversed the Matrigel to the lower surface of the insert were fixed using $4 \%$ paraformaldehyde at room temperature for $20 \mathrm{~min}$ and stained with eosin $(0.5 \%$, R20593, Shanghai Yuan Ye Biological Technology Co., Ltd.) (SKOV3 cells) or hematoxylin (D005, Nanjing Jiancheng Bioengineering Institute, Nanjing China, $0.5 \%$ ) (OVCAR3 cells) alone, both at room temperature for $20 \mathrm{~min}$. Cells were observed and calculated in five random fields using alight microscope (magnification x100).

Statistical analysis. All statistical analyses were performed using GraphPad Prism (version 5.0; GraphPad Software, Inc., La Jolla, CA, USA). The data are presented as the mean \pm standard error of the mean. Statistically significant differences between mean values of two groups were identified using unpaired Student's t-test. Statistically significant differences between mean values of $\geq 3$ groups were identified using analysis of variance and Student-Newman-Keuls post-hoc test. $\mathrm{P}<0.05$ was considered to indicate a statistically significant difference. 


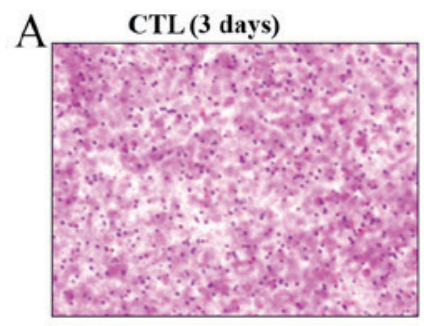

CTL (3 days)
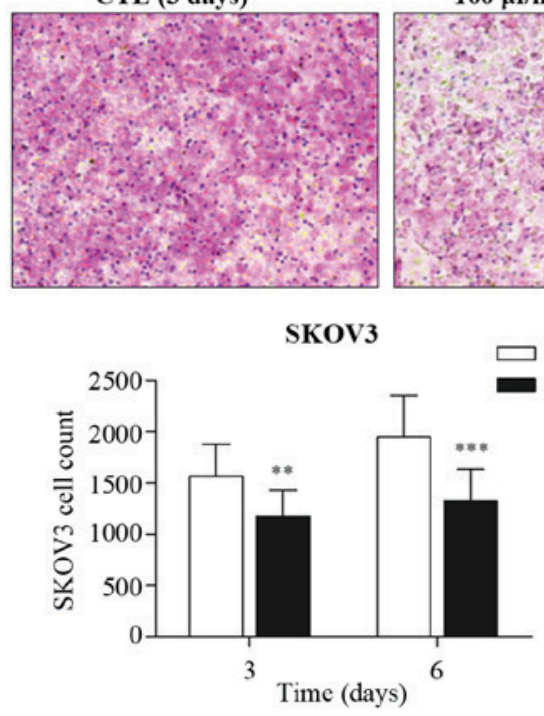

$100 \mu \mathrm{l} / \mathrm{ml}$ VitD (3 days)

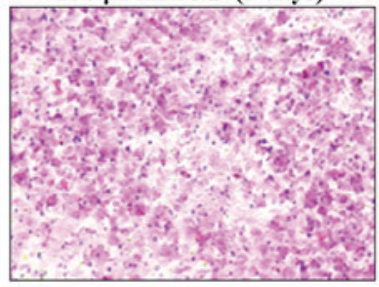

$100 \mu \mathrm{l} / \mathrm{ml}$ VitD (6 days)

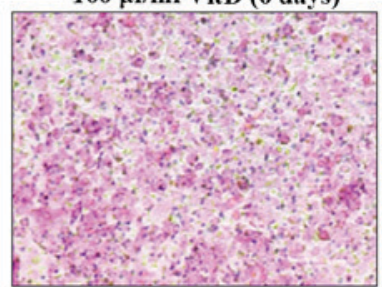

CTL

$100 \mu \mathrm{l} / \mathrm{ml}$ VitD

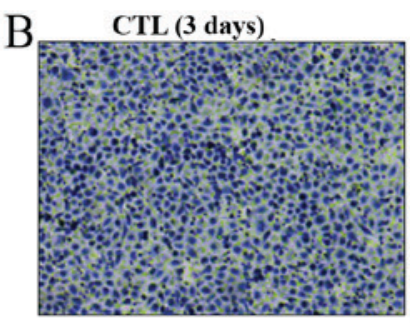

CTL (3 days)

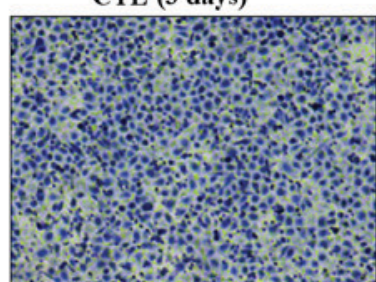

OVCAR3

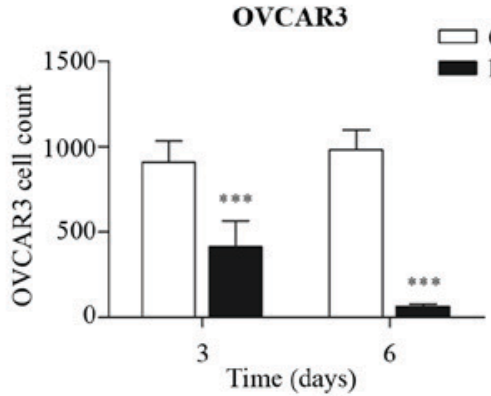

$100 \mu \mathrm{l} / \mathrm{ml}$ VitD (3 days)

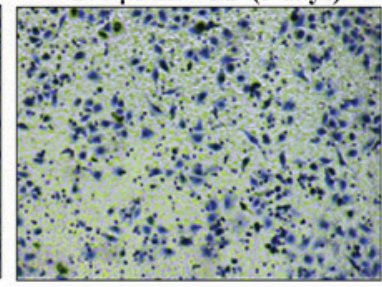

$100 \mu \mathrm{l} / \mathrm{ml}$ VitD (6 days)

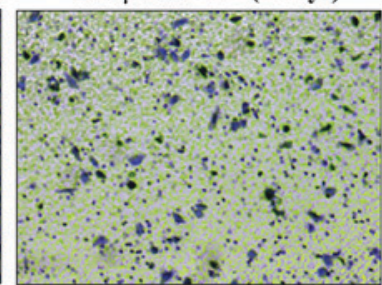

$\square$ CTL

$100 \mu \mathrm{l} / \mathrm{ml}$ VitD

Figure 2. Active vitamin D inhibits the invasive ability of SKOV3 and OVCAR3 cells. (A) SKOV3 cells and (B) OVCAR3 cells were treated with $100 \mu 1 / \mathrm{m}$ active vitamin $\mathrm{D}$ for 3 or 5 days in a transwell invasion assay. ${ }^{* *} \mathrm{P}<0.01,{ }^{* * * *} \mathrm{P}<0.001$, compared with control. CTL, control; VitD, active vitamin D.

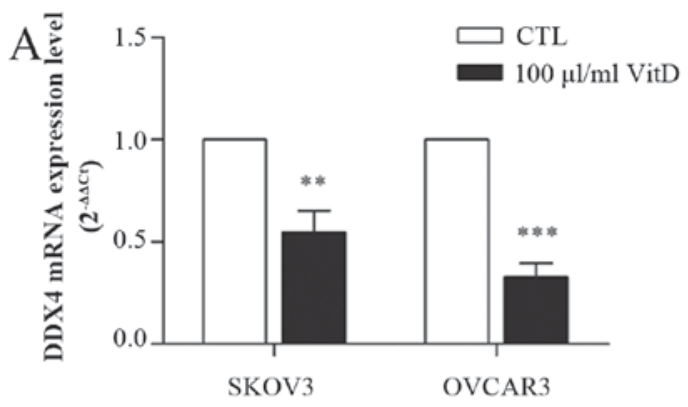

B

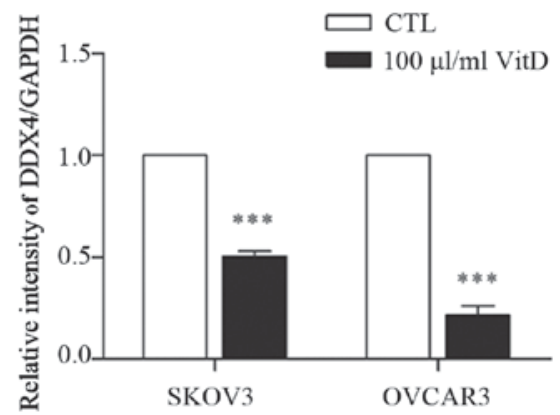

Figure 3. Active vitamin D reduces the expression level of DDX4 in SKOV3 and OVCAR3 cells. SKOV3 or OVCAR3 cells were treated with $100 \mu \mathrm{l} / \mathrm{ml}$ active vitamin D. (A) Reverse transcription-quantitative polymerase chain reaction analysis, and (B) western blot analysis was performed to examine the expression level of DDX4. ${ }^{* *} \mathrm{P}<0.01,{ }^{* * *} \mathrm{P}<0.001$, compared with control. DDX4, DEAD-box helicase 4; CTL, control; VitD, vitamin D.

\section{Results}

Active vitamin $D$ suppresses the proliferation of ovarian cancer cells. To evaluate the effect of active vitamin D on ovarian cancer, SKOV3 or OVCAR3 cells were treated with varying concentrations of biologically active vitamin $\mathrm{D}$ for $72 \mathrm{~h}$. The relative cell number was quantified using CCK-8. It was demonstrated that $100 \mu \mathrm{l} / \mathrm{ml}$ active vitamin D was able to 
significantly inhibit the proliferation of SKOV3 and OVCAR3 cells, and a further increase in concentration caused a greater inhibitory effect (Fig. 1).

Active vitamin D inhibits the invasion of ovarian cancer cells. Active vitamin D treatment reduced the number of SKOV3 or OVCAR3 cells able to migrate to the lower surface of transwell inserts (Fig. 2A and B), suggesting that vitamin D could partially block the invasion ability of ovarian cancer cells.

Active vitamin D downregulated the expression of DDX4 in ovarian cancer cells. It has been established that DDX4 is overexpressed in epithelial ovarian cancer and can be used as an ovarian cancer stem cell marker (14). To assess whether vitamin D affects the expression of DDX4, SKOV3 or OVCAR3 cells were treated with $100 \mu \mathrm{l} / \mathrm{ml}$ active vitamin D for $72 \mathrm{~h}$ prior to RT-qPCR and western blot analyses. It was demonstrated that vitamin $\mathrm{D}$ treatment downregulated the expression of DDX4 at the mRNA (Fig. 3A) and protein (Fig. 3B) levels.

DDX4 knockdown inhibits invasion of ovarian cancer cells. To investigate whether DDX4 could influence the invasion of ovarian cancer cells, several independent siRNAs targeting DDX4 were transfected into SKOV3and OVCAR3 cells individually. The knockdown efficiency was evaluated by RT-qPCR, which demonstrated that transfection with siDDX4-121 or siDDX4-712 could significantly reduce

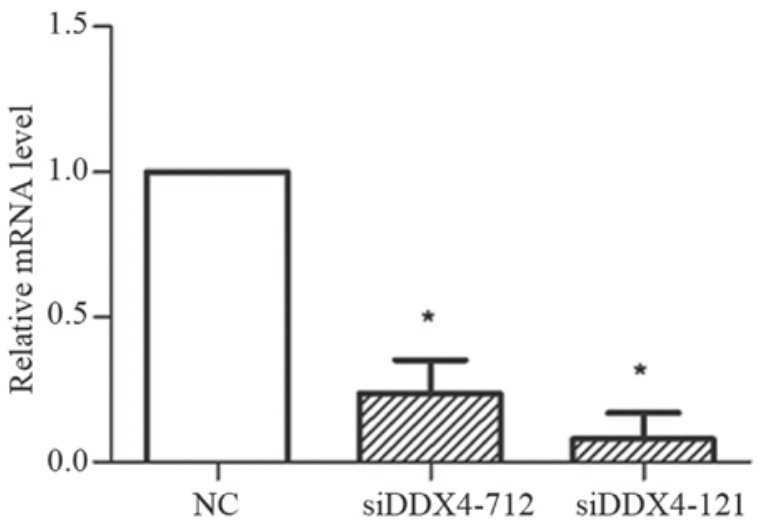

Figure 4. DDX4 siRNA reduces the expression level of DDX4. SKOV3 or OVCAR3 cells were transfected with two independent siRNAs against DDX4, and the DDX4 mRNA level was examined by reverse transcription-quantitative polymerase chain reaction, relative to GADPH as an internal control. ${ }^{*} \mathrm{P}<0.05$, compared with blank control group. DDX4, DEAD-box helicase 4; siRNA, small interfering RNA; NC, negative control.

DDX4 expression (Fig. 4). siDDX4-121-transfected cells were selected for the transwell assay, due to the greater inhibition of DDX4 expression achieved using this siRNA. Invasive cells were stained 3 or 5 days subsequent to siRNA treatment (Fig. 5A and B). DDX4 knockdown partially inhibited the invasive ability of SKOV3 and OVCAR3 cells (Fig. 5A and B).
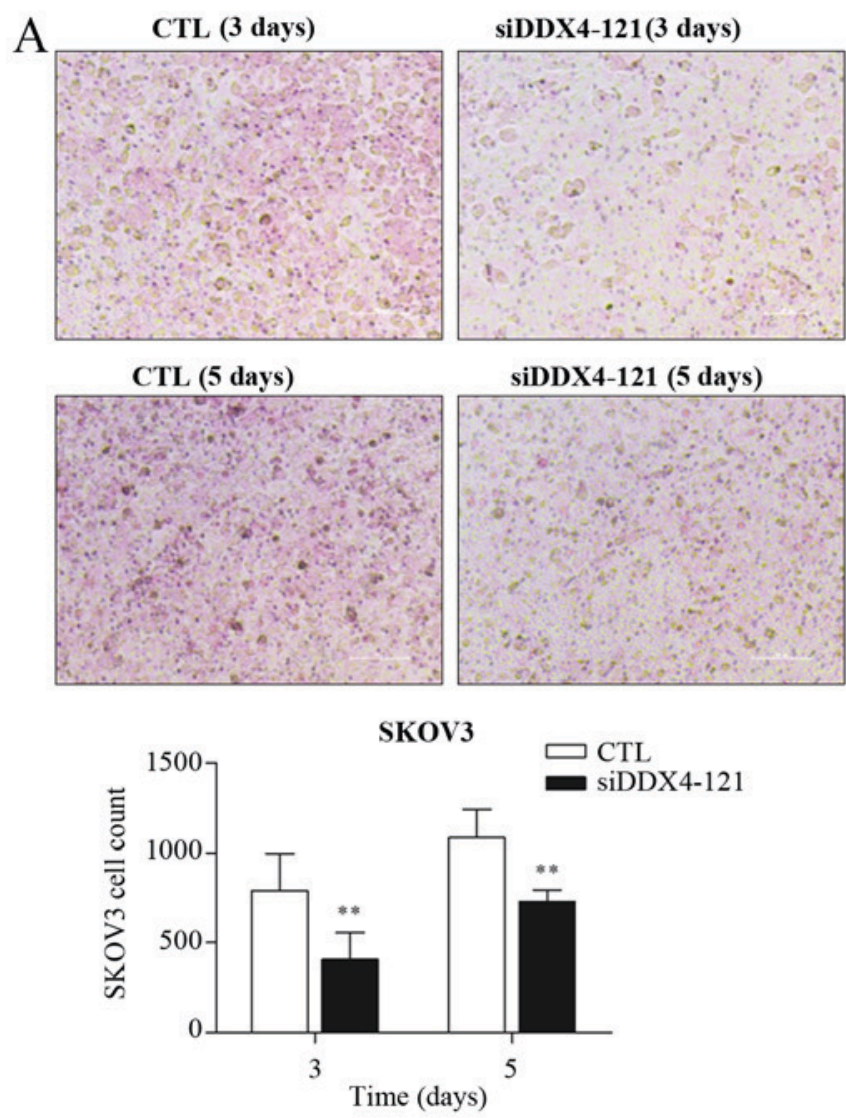

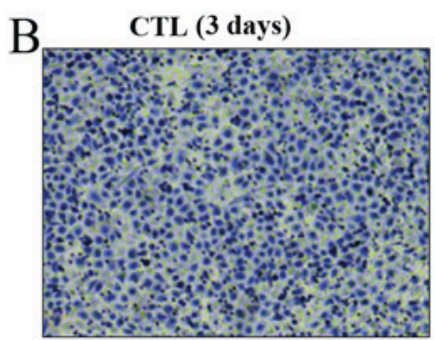

siDDX4-121 ( 3 days)
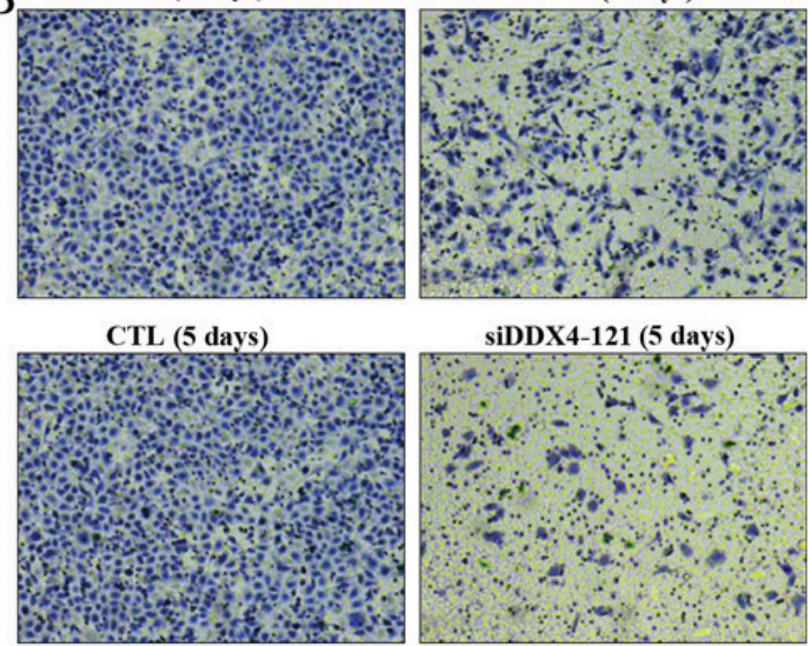

siDDX4-121 (5 days)

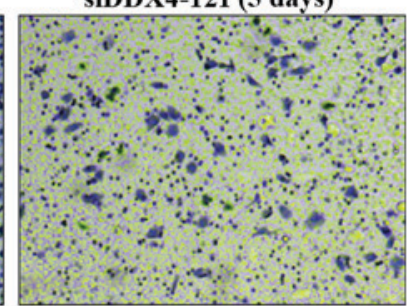

OVCAR3

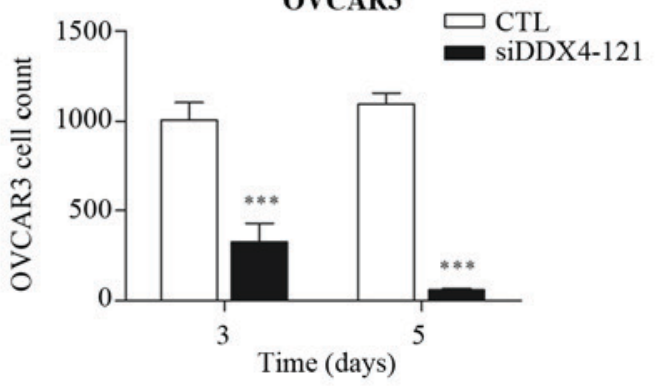

Figure 5. DDX4 knockdown inhibited the invasive ability of SKOV3 and OVCAR3 cells. (A) SKOV3 cells or (B) OVCAR3 cells were treated with siDDX4-121 for 3 or 5 days in a transwell assay. ${ }^{* *} \mathrm{P}<0.01,{ }^{* * *} \mathrm{P}<0.001$, compared with control. DDX4, DEAD-box helicase 4 ; siDDX4, small interfering RNA targeting DDX4. 


\section{Discussion}

Vitamin D was first recognized for its regulatory function in calcium-phosphorus balance (15). Recently, it has been demonstrated that active vitamin D affects various cellular processes, including proliferation, invasion, differentiation and malignant transformation $(8,16-18)$ in multiple types of cancer $(18,19)$, including male reproductive system carcinomas and prostate cancer (12). It has also been suggested that vitamin D could prevent ovarian cancer progression (8). The present study demonstrated that the proliferative and invasive abilities of SKOV3 and OVCAR3 ovarian cancer cells could be inhibited by active vitamin D. However, the molecular mechanisms of how vitamin D inhibits the proliferation and invasion of ovarian cancer cells remain to be further investigated.

DDX4 is expressed exclusively in the ovaries and testes. The expression level of DDX4 in SKOV3 and OVCAR3 cells was downregulated by active vitamin $\mathrm{D}$ at the mRNA and protein level. Knockdown of DDX4 by siRNA partially inhibited the invasion ability of SKOV3 and OVCAR3 cells. It is speculated that vitamin D may inhibit the invasion of ovarian cancer cells through downregulating the expression of DDX4.

In conclusion, vitamin $\mathrm{D}$ treatment reduced the proliferation and invasion of ovarian cancer cells. DDX4, which was previously found to be overexpressed in ovarian cancer, was downregulated by vitamin D on the mRNA and protein levels. DDX4 knockdown also inhibited the invasion of ovarian cancer cells. Therefore, the use of vitamin D should be considered as a potential novel therapy for ovarian cancer.

\section{Acknowledgements}

Not applicable.

\section{Funding}

The present was supported by the National Natural Science Foundation of China (grant nos. 81672560 and 31501137), and Jiangsu Basic Research Funds for Young Scientists (grant no. BK20150351).

\section{Availability of data and materials}

The analyzed data sets generated during the study are available from the corresponding author upon request.

\section{Authors' contributions}

All authors have read and approved the manuscript. ZS performed the experiment and analyzed the data. JX, PW, JT, XS and JL contributed todatacollectionandanalysis. YC,FR, andLXdesigned the study and wrote the manuscript.

\section{Ethics approval and consent to participate}

Not applicable.

\section{Consent for publication}

Not applicable.

\section{Competing interests}

The authors declare that they have no competing interests.

\section{References}

1. Lim HJ and Ledger W: Targeted therapy in ovarian cancer. Womens Health (Lond) 12: 363-378, 2016.

2. Clarke-Pearson DL: Clinical practice. Screening for ovarian cancer. N Engl J Med 361: 170-177, 2009.

3. Jelovac D and Armstrong DK: Recent progress in the diagnosis and treatment of ovarian cancer. CA Cancer J Clin 61: 183-203, 2011.

4. Guenther J, Stiles A and Champion JD: The lived experience of ovarian cancer: A phenomenological approach. J Am Acad Nurse Pract 24: 595-603, 2012.

5. Yahara K, Ohguri T, Imada H, Yamaguchi S, Kawagoe T, Matsuura Y, Hachisuga T and Korogi Y: Epithelial ovarian cancer: Definitive radiotherapy for limited recurrence after complete remission had been achieved with aggressive front-line therapy. J Radiat Res 54: 322-329, 2013.

6. Baruah U, Barmon D, Kataki AC, Deka P, Hazarika M and Saikia BJ: Neoadjuvant chemotherapy in advanced epithelial ovarian cancer: A survival study. Indian J Med Paediatr Oncol 36: 38-42, 2015.

7. Wahler J, So JY, Cheng LC, Maehr H, Uskokovic M and Suh N: Vitamin D compounds reduce mammosphere formation and decrease expression of putative stem cell markers in breast cancer. J Steroid Biochem Mol Biol 148: 148-155, 2015.

8. So JY and Suh N: Targeting cancer stem cells in solid tumors by vitamin D. J Steroid Biochem Mol Biol 148: 79-85, 2015.

9. Garland CF, Mohr SB, Gorham ED, Grant WB and Garland FC: Role of ultraviolet $B$ irradiance and vitamin $D$ in prevention of ovarian cancer. Am J Prev Med 31: 512-514, 2006.

10. Granato T, Manganaro L, Petri L, Porpora MG, Viggiani V, Angeloni A and Anastasi E: Low 25-OH vitamin D levels at time of diagnosis and recurrence of ovarian cancer. Tumour Biol 37: 2177-2181, 2016

11. Castrillon DH, Quade BJ, Wang TY, Quigley C and Crum CP: The human VASA gene is specifically expressed in the germ cell lineage. Proc Natl Acad Sci USA 97: 9585-9590, 2000.

12. Hashimoto H, Sudo T, Mikami Y, Otani M, Takano M, Tsuda H, Itamochi H, Katabuchi H, Ito M and Nishimura R: Germ cell specific protein VASA is over-expressed in epithelial ovarian cancer and disrupts DNA damage-induced G2 checkpoint. Gynecol Oncol 111: 312-319, 2008.

13. Livak KJ and Schmittgen TD: Analysis of relative gene expression data using real-time quantitative PCR and the 2(-Delta Delta C(T)) method. Methods 25: 402-408, 2001.

14. Kim KH, Kang YJ, Jo JO, Ock MS, Moon SH, Suh DS, Yoon MS, Park ES, Jeong N, Eo WK, et al: DDX4 (DEAD box polypeptide 4) colocalizes with cancer stem cell marker CD133 in ovarian cancers. Biochem Biophys Res Commun 447: 315-322, 2014.

15. Tavani A, Bertuccio P, Bosetti C, Talamini R, Negri E, Franceschi S, Montella M and La Vecchia C: Dietary intake of calcium, vitamin D, phosphorus and the risk of prostate cancer. Eur Urol 48: 27-33, 2005.

16. Wang WL,WelshJ and TenniswoodM:1,25-DihydroxyvitaminD3 modulates lipid metabolism in prostate cancer cells through miRNA mediated regulation of PPARA. J Steroid Biochem Mol Biol 136: 247-251, 2013.

17. Wang WL, Chatterjee N, Chittur SV, Welsh J and Tenniswood MP: Effects of 1 $\alpha, 25$ dihydroxyvitamin D3 and testosterone on miRNA and mRNA expression in LNCaP cells. Mol Cancer 10: 58, 2011.

18. Thorne $J$ and Campbell MJ: The vitamin D receptor in cancer. Proc Nutr Soc 67: 115-127, 2008.

19. Giovannucci E: Expanding roles of vitamin D. J Clin Endocrinol Metab 94: 418-420, 2009.

This work is licensed under a Creative Commons Attribution-NonCommercial-NoDerivatives 4.0 International (CC BY-NC-ND 4.0) License. 